\title{
Modification of surgical technique for ascending aortic atherosclerosis: Impact on stroke reduction in coronary artery bypass grafting
}

\author{
Herbert Bernd Hangler, MD \\ Georg Nagele, MD \\ Michael Danzmayr, MD \\ Ludwig Mueller, MD \\ Elfriede Ruttmann, MD \\ Guenther Laufer, MD \\ Johannes Bonatti, MD
}

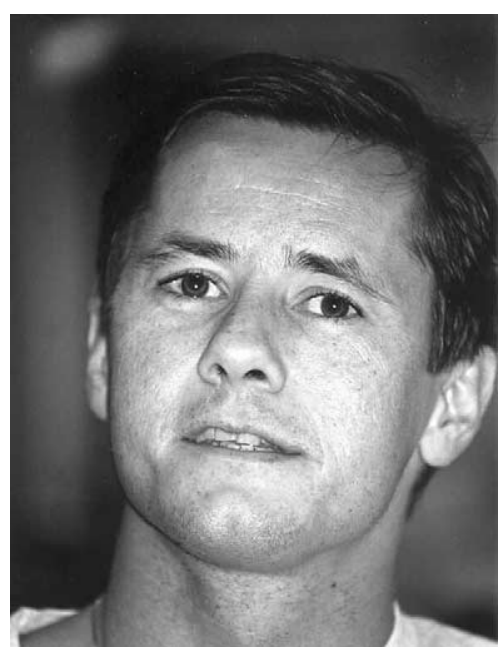

Dr Hangler

From the Department of Cardiac Surgery, Innsbruck University Hospital, Innsbruck, Austria.

Received for publication July 24, 2002; revisions requested Sept 10, 2002; revisions received Oct 28, 2002; accepted for publication Nov 1, 2002.

Address for reprints: Herbert Bernd Hangler, MD, Department of Cardiac Surgery, Innsbruck University Hospital, Anichstrasse 35, 6020, Innsbruck, Austria (E-mail: herbert.hangler@uibk.ac.at).

J Thorac Cardiovasc Surg 2003;126: 391-400

Copyright $\odot 2003$ by The American Association for Thoracic Surgery

$0022-5223 / 2003 \$ 30.00+0$

doi:10.1016/S0022-5223(03)00395-7
Objective: Use of epiaortic scanning in coronary surgery is still a matter of debate. It is unclear whether the findings obtained by epiaortic scanning lead to effective changes in surgical technique that may reduce stroke rates.

Methods: Epiaortic scanning was performed in 352 patients undergoing primary coronary artery bypass grafting before opening the pericardium using a $7.5-\mathrm{MHz}$ ultrasonic probe. In the presence of moderate atherosclerosis (maximum aortic wall thickness of 3 to $5 \mathrm{~mm}$ ), primarily single aortic crossclamping was carried out. In cases of severe sclerosis (maximum aortic wall thickness $>5 \mathrm{~mm}$ ), aortic no-touch techniques on the beating heart were used.

Results: The degree of ascending aortic atherosclerosis was normal or mild in 151 patients $(42.9 \%)$, moderate in 167 patients $(47.5 \%)$, and severe in 34 patients (9.6\%). The operative technique was modified in $31.1 \%$ of patients with moderate aortic sclerosis and in $91.2 \%$ of patients with severe aortic sclerosis. Perioperative mortality was $0.0 \%$ for mild disease, $3.0 \%$ for moderate disease, and $8.8 \%$ for severe disease $(P=.005)$. Corresponding stroke rates reached $2.0 \%, 2.4 \%$, and 2.9\% $(P=.935)$. Logistic regression adjusting for EuroSCORE showed that ascending aortic atherosclerosis was an independent predictor of perioperative mortality $(P=.013$, odds ratio 1.67 , confidence interval $1.11-2.50)$. The influence of aortic disease on the stroke prevalence was probably due to chance $(P=.935)$, demonstrating a potentially positive effect of operative modifications concerning stroke caused by aortic manipulation.

Conclusions: We conclude that intraoperative screening of coronary artery bypass grafting patients by epiaortic scanning can reveal useful information about the operative risk and with an aortic no-touch concept, perioperative stroke rates in high-risk patients may be lower than predicted.

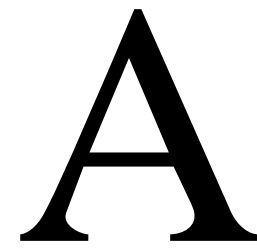

therosclerosis of the ascending aorta has emerged as one of the most important risk factors for postoperative complications in cardiac surgery, particularly in on-pump coronary artery bypass grafting $(\mathrm{CABG})$ when the diseased aorta is manipulated by cannulation and clamping. These maneuvers can be associated with intraoperative atheromatous embolization into the cerebral circulation, resulting in persistent cognitive deficit or postoperative 


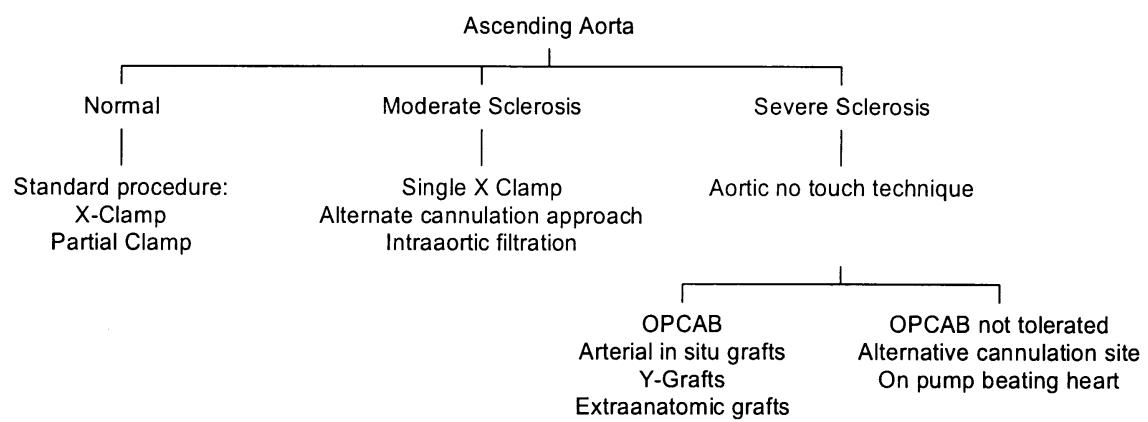

Figure 1. Algorithm for decision making with ascending aortic atherosclerotic disease.

stroke, a serious complication with a considerable mortality of up to $21 \% .^{1}$ Furthermore, embolization of atheromatous debris from atherosclerotic ascending aortic lesions into the coronary microcirculation accounts, at least in part, for the prevalence of perioperative myocardial infarction. ${ }^{2}$ In addition, atherosclerosis of the ascending aorta has been found to be an independent risk factor for long-term neurologic events and mortality in patients having undergone cardiac surgery. ${ }^{3,4}$ The current trend is toward surgical treatment of coronary heart disease in older patients with a higher comorbidity. In addition, the severity of ascending aortic atherosclerotic disease and the stroke rate are known to increase with age. ${ }^{5,6}$ Epiaortic ultrasonography has been added to the armament of cardiac surgeons as a fast, noninvasive, and sensitive technique that provides information of the ascending aortic wall in its entire length and circumference and seems to be superior to transesophageal echocardiography. ${ }^{7}$ Furthermore, epiaortic scanning (EAS) is more accurate than palpation in identifying mobile atheroma in the ascending aortic lumen. The aim of our study was to evaluate the postoperative short- and medium-term outcome in patients with ascending aortic atherosclerosis detected by intraoperative epiaortic ultrasound. According to an algorithm, taking into account the severity of the aortic disease, the standard surgical technique was modified. It was another aim of the study to assess risk groups for the presence of ascending aortic atherosclerosis and to evaluate its role as a predictor for adverse postoperative events.

\section{Patients and Methods \\ Patient Population}

The study population consisted of 352 consecutive patients undergoing isolated primary $\mathrm{CABG}$ and intraoperative evaluation of the ascending aorta with epiaortic ultrasound at Innsbruck University Hospital from April 1998 to October 2001. These patients represented $40 \%$ of all primary CABG procedures at our institution during this time frame.

\section{Informed Consent}

Written informed consent for coronary artery bypass grafting was obtained from all patients preoperatively and information about potential operative modifications in risk groups was selectively provided.

\section{Definition of End Points}

Perioperative myocardial infarction: Creatine kinase $\mathrm{MB}$ of at least $50 \mathrm{IU} / \mathrm{mL}$ or presence of a new pathologic $\mathrm{Q}$ wave on the electrocardiogram within 30 days or during the same hospital stay.

Perioperative stroke: Any new focal neurologic deficit or disturbance of consciousness lasting more than 24 hours, confirmed by cerebral computed tomographic scan, within 30 days or during the same hospital stay.

Perioperative death: All-cause mortality within 30 days or during the same hospital stay.

\section{Palpation of the Ascending Aorta}

Palpation of the ascending aorta for identification of calcified areas was done with caution to avoid dislodgment of embolic material.

\section{Ultrasonic Methods}

EAS was performed with a $7.5-\mathrm{MHz}$ linear ultrasonic probe (Hewlett-Packard Image Point HX; Hewlett-Packard Company, Andover, Mass) coated with ultrasonic gel as an acoustic medium, packed in a sterile plastic sleeve. EAS was performed before opening the pericardium to sustain an echolucent window for optimal visualization of the anterior ascending aortic wall. The probe was then manipulated gently to obtain longitudinal and transverse views from the aortic valve up to the innominate artery. Ascending aortic atherosclerotic disease (AAAD) was defined as normal/mild (aortic wall $\leq 3 \mathrm{~mm}$ ), moderate (aortic wall 3 to 5 $\mathrm{mm}$ ), and severe (aortic wall thickness $>5 \mathrm{~mm}$ and/or the presence of marked calcification, protruding or mobile intraluminal atheromatous portions, and ulcerated plaques) according to the classification reported by Wareing and colleagues. ${ }^{6}$

Carotid artery Doppler and duplex ultrasound are routinely performed in our patients over 40 years of age. Carotid artery stenosis was graded as insignificant (luminal narrowing $\leq 50 \%$ ), moderate (luminal narrowing 50\%-79\%), severe (luminal narrowing $\geq 80 \%$ or complete occlusion). Cerebrovascular disease was 
TABLE 1. Baseline patient characteristics according to the severity of ascending aorta atherosclerosis

\begin{tabular}{|c|c|c|c|c|c|}
\hline & $\begin{array}{c}\text { All patients } \\
\text { (n = 352) }\end{array}$ & $\begin{array}{c}\text { Normal/mild } \\
\text { (n = 151) }\end{array}$ & $\begin{array}{l}\text { Moderate } \\
(n=167)\end{array}$ & $\begin{array}{c}\text { Severe } \\
(\mathrm{n}=34)\end{array}$ & $P$ value \\
\hline Age (years) & $65.6 \pm 9.3$ & $62.7 \pm 9.7$ & $67.2 \pm 8.5$ & $70.4 \pm 7.3$ & $<.001$ \\
\hline$>70$ years of age & $122(34.7 \%)$ & $37(24.5 \%)$ & $63(37.7 \%)$ & $22(64.7 \%)$ & $<.001$ \\
\hline Male & $269(76.4 \%)$ & $109(72.2 \%)$ & $131(78.4 \%)$ & $29(85.3 \%)$ & .186 \\
\hline BMI & $26.5 \pm 3.5$ & $26.9 \pm 3.6$ & $26.2 \pm 3.2$ & $25.9 \pm 3.8$ & .152 \\
\hline Hypertension & $269(76.4 \%)$ & $106(70.2 \%)$ & $134(80.2 \%)$ & $29(85.3 \%)$ & .048 \\
\hline Diabetes mellitus & $87(24.7 \%)$ & $37(24.5 \%)$ & $42(25.1 \%)$ & $8(23.5 \%)$ & .977 \\
\hline CVD & $79(22.4 \%)$ & $27(17.9 \%)$ & $33(19.8 \%)$ & $19(55.9 \%)$ & $<.001$ \\
\hline History of neurologic events & $27(7.7 \%)$ & $8(5.3 \%)$ & $16(9.6 \%)$ & $3(8.8 \%)$ & .346 \\
\hline PVD & $62(17.6 \%)$ & $13(8.6 \%)$ & $32(19.2 \%)$ & $17(50.0 \%)$ & $<.001$ \\
\hline Extracardiac atherosclerosis & $113(32.1 \%)$ & $33(21.9 \%)$ & $55(32.9 \%)$ & $25(73.5 \%)$ & $<.001$ \\
\hline COPD & $32(9.1 \%)$ & $11(7.3 \%)$ & $15(9.0 \%)$ & $6(17.6 \%)$ & .164 \\
\hline Atrial fibrillation & $13(3.7 \%)$ & $3(2.0 \%)$ & $9(5.4 \%)$ & $1(2.9 \%)$ & .065 \\
\hline NYHA & $2.5 \pm 1.0$ & $2.4 \pm 1.0$ & $2.5 \pm 1.0$ & $2.9 \pm 1.1$ & .426 \\
\hline Unstable angina & $66(18.8 \%)$ & $29(19.2 \%)$ & $27(16.2 \%)$ & $10(29.4 \%)$ & .193 \\
\hline Emergent surgery & $10(2.8 \%)$ & $5(3.3 \%)$ & $3(1.8 \%)$ & $2(5.9 \%)$ & .383 \\
\hline Creatinine preoperative & $1.16 \pm 0.48$ & $1.09 \pm 0.23$ & $1.20 \pm 0.62$ & $1.33 \pm 0.43$ & .012 \\
\hline EF $(\%)$ & $58.1 \pm 12.7$ & $59.4 \pm 13.0$ & $57.7 \pm 12.3$ & $54.5 \pm 13.3$ & .104 \\
\hline EuroSCORE & $4.3 \pm 3.2$ & $3.5 \pm 2.7$ & $4.6 \pm 3.3$ & $6.4 \pm 3.1$ & $<.001$ \\
\hline McSPI probability & $2.6 \pm 3.2$ & $1.8 \pm 1.9$ & $3.0 \pm 3.8$ & $4.4 \pm 3.3$ & $<.001$ \\
\hline
\end{tabular}

$B M I$, Body mass index; creatinine, serum creatinine $\mathrm{mg} / \mathrm{dL} ; E F$, ejection fraction; $C O P D$, chronic obstructive pulmonary disease; $C V D$, cerebrovascular disease; PVD, peripheral vascular disease; NYHA, New York Heart Association; McSPI, Multicenter Study of Perioperative Ischemia.

TABLE 2. Intraoperative patient characteristics

\begin{tabular}{|c|c|c|c|c|c|}
\hline & $\begin{array}{l}\text { All patients } \\
(\mathrm{n}=352)\end{array}$ & $\begin{array}{c}\text { Normal/mild } \\
(\mathrm{n}=151)\end{array}$ & $\begin{array}{l}\text { Moderate } \\
(n=167)\end{array}$ & $\begin{array}{c}\text { Severe } \\
(n=34)\end{array}$ & $P$ value \\
\hline Ascending aortic maximal wall thickness $(\mathrm{mm})$ & $3.4 \pm 1.3$ & $2.4 \pm 0.4$ & $3.7 \pm 0.6$ & $6.1 \pm 1.5$ & $<.001$ \\
\hline Number of distal anastomoses & $3.1 \pm 0.8$ & $3.1 \pm 0.8$ & $3.2 \pm 0.8$ & $2.7 \pm 0.7$ & .251 \\
\hline Number of central anastomoses & $2.0 \pm 0.8$ & $2.0 \pm 0.8$ & $2.1 \pm 0.7$ & $1.7 \pm 0.7$ & .146 \\
\hline AAAD detected on palpation & $86(25.7 \%)$ & $15(10.6 \%)$ & $41(25.6 \%)$ & $30(90.9 \%)$ & $<.001$ \\
\hline Partial clamp & $276(78.4 \%)$ & $143(94.7 \%)$ & $127(76.0 \%)$ & $6(17.6 \%)$ & $<.001$ \\
\hline Operative modifications & $90(25.6 \%)$ & $7(4.6 \%)$ & $52(31.1 \%)$ & $31(91.2 \%)$ & $<.001$ \\
\hline Alternate cannulation site & $24(6.8 \%)$ & $4(2.6 \%)$ & $15(9.0 \%)$ & $5(14.7 \%)$ & .013 \\
\hline Alternate clamp site & $8(2.3 \%)$ & 0 & $5(3.0 \%)$ & $3(8.8 \%)$ & .005 \\
\hline Single $X$ clamp & $36(10.2 \%)$ & $1(0.7 \%)$ & $32(19.2 \%)$ & $3(8.8 \%)$ & $<.001$ \\
\hline Beating heart & $33(9.4 \%)$ & $2(1.3 \%)$ & $6(3.6 \%)$ & $25(73.5 \%)$ & $<.001$ \\
\hline Extraanatomic graft & $29(8.2 \%)$ & $2(1.3 \%)$ & $4(2.4 \%)$ & $23(67.6 \%)$ & $<.001$ \\
\hline
\end{tabular}

$A A A D$, Ascending aortic atherosclerotic disease.

defined as the presence of moderate to severe carotid artery stenosis.

\section{Follow-up}

Patients or their family physicians completed a telephone questionnaire to identify those who had had a major adverse neurologic event such as stroke or transient ischemic attack as well as cardiac events such as myocardial infarction and coronary reintervention or death. The grade of activity in patients who had a perioperative stroke was evaluated according to the Barthel Index. Follow-up was available in $90.0 \%$ of all patients, in $88.1 \%$ of no/mild AAAD, in $93.4 \%$ of moderate AAAD, and in $91.2 \%$ of severe AAAD.

\section{Surgical Technique}

The standard procedure for coronary artery revascularization in patients with multivessel disease at our institution is extracorporeal circulation, moderate hypothermia $\left(32^{\circ} \mathrm{C}\right)$, and antegrade-retrograde delivery of cold cardioplegic solution. Aortic anastomoses of the grafts are usually performed with a partial occluding clamp. When the surgeon encountered AAAD by EAS, operative modifications were adopted according to an algorithm taking into account the severity of the aortic disease (Figure 1).

Single crossclamp technique. After all the distal anastomoses were done, retrograde cardioplegia was administered and the proximal anastomoses were performed with the aortic clamp on, with running 6-0 polypropylene sutures. Before the last proximal anastomosis was completed, the ascending aorta was deaired by application of retrograde cardioplegia. 8,9

Aortic no-touch surgery. When severe disease of the ascending aorta was diagnosed, the main objective was to avoid any manipulation of the aorta. Therefore, off-pump coronary surgery was preferred whenever possible. Both internal thoracic arteries 
TABLE 3. Perioperative outcome according to the severity of ascending aortic atherosclerotic disease

\begin{tabular}{|c|c|c|c|c|c|}
\hline & $\begin{array}{l}\text { All patients } \\
\text { (n = 352) }\end{array}$ & $\begin{array}{c}\text { Normal mild } \\
(\mathrm{n}=151)\end{array}$ & $\begin{array}{l}\text { Moderate } \\
(n=167)\end{array}$ & $\begin{array}{l}\text { Severe } \\
(n=34)\end{array}$ & $P$ value \\
\hline Stroke & $8(2.3 \%)$ & $3(2.0 \%)$ & $4(2.4 \%)$ & $1(2.9 \%)$ & .935 \\
\hline Death & $8(2.3 \%)$ & 0 & $5(3.0 \%)$ & $3(8.8 \%)$ & .005 \\
\hline Myocardial infarction & $14(3.7 \%)$ & $3(2.0 \%)$ & $5(3.0 \%)$ & $5(14.7 \%)$ & .001 \\
\hline Postoperative AF & $115(32.7 \%)$ & $48(31.8 \%)$ & $52(31.1 \%)$ & $15(44.1 \%)$ & .323 \\
\hline
\end{tabular}

$A F$, Atrial fibrillation.

TABLE 4. Logistic regression, Cox regression

\begin{tabular}{|c|c|c|c|c|c|c|c|c|c|c|c|c|}
\hline \multirow[b]{2}{*}{ Variable } & \multicolumn{9}{|c|}{ Perioperative } & \multicolumn{3}{|c|}{$\begin{array}{c}\text { 36-month } \\
\text { myocardial infarction }\end{array}$} \\
\hline & $P$ & OR & $95 \% \mathrm{Cl}$ for $\mathrm{OR}$ & $P$ & OR & $95 \% \mathrm{Cl}$ for $\mathrm{OR}$ & $P$ & HR & 95\% Cl for HR & $P$ & HR & 95\% Cl for HR \\
\hline EuroSCORE & .0014 & 1.5006 & $1.1708-1.9233$ & .0009 & 1.3509 & $1.1311-1.6133$ & $<.0001$ & 1.4056 & $1.2171-1.6232$ & .0005 & 1.2758 & $1.1118-1.4640$ \\
\hline $\begin{array}{l}\text { Ascending aorta } \\
\text { maximal wall } \\
\text { thickness }(\mathrm{mm})\end{array}$ & .0130 & 1.6681 & $1.1141-2.4978$ & .0452 & 1.4107 & $1.0074-1.9755$ & .0282 & 1.3511 & $1.0326-1.7678$ & .0938 & 1.2718 & $.9600-1.6849$ \\
\hline
\end{tabular}

$O R, 0$ dds ratio; $H R$, hazard ratio.

were used either as in situ grafts or, when the right internal thoracic artery was too short to reach the target coronary, it was anastomosed to the left internal thoracic artery as a Y graft. For complete revascularization, vein and or radial artery grafts were also implanted into the internal thoracic arteries as Y grafts. When the internal thoracic arteries were considered too small as an inflow source, the axillary or innominate arteries were preferred as an extra-anatomic site for graft inflow. ${ }^{10}$

\section{Data Analysis}

Data were collected in Excel 5.0 for Windows. Data analysis was performed with SPSS 9.0 for Windows (SPSS, Inc, Chicago, Ill). Continuous variables were expressed as means \pm SDs and categorical variables as absolute numbers (percentages).

Univariable analysis. Dichotomous variables were compared with chi-square test, continuous variables with analysis of variance. Kaplan-Meier survival analysis was used to describe the time to the first event (stroke, myocardial infarction, and all-cause mortality), combined with a log-rank test. In all nonfatal events, data were censored at the time of death if the event of interest had not previously occurred.

Multivariable analysis. Logistic regression analyses for the perioperative end points mortality and myocardial infarction and Cox proportional hazard regression analyses for the medium-term end point mortality and myocardial infarction were performed, adjusting ascending aorta maximal wall thickness (millimeters) for EuroSCORE.

The cumulative risk-adjusted mortality (CRAM) depicts the lives saved or lost versus EuroSCORE predictions, ${ }^{11}$ adding value if a patient survived the hospital stay (eg, +0.05 if a patient with $5 \%$ predicted risk survived) and subtracting value if a patient died during hospital stay (eg, -0.95 if a patient with $5 \%$ predicted risk died). If the line equals the baseline, then the observed risk equals the predicted risk; if the line moves above the baseline, then the observed result expresses the number of lives gained versus the
EuroSCORE. The horizontal axis depicts the patient numbers in a chronological order plotted from left to right. Cumulative riskadjusted stroke prevalence (CRAS) follows the same principles using the Multicenter Study of Perioperative Ischemia (McSPI) stroke risk index as the predicting tool. ${ }^{12}$ The McSPI stroke risk index was converted to probability of event by logistic transformation. The plots were created with Excel 5.0 for Windows.

The McSPI developed, validated, and published a predictive stroke risk index for patients undergoing isolated CABG using conventional cardiopulmonary bypass for support. The McSPI stroke risk index is intended to be applied preoperatively to estimate the likelihood that a patient will experience a major perioperative neurologic event.

\section{Results}

The grade of ascending aortic atherosclerosis was normal or mild in 151 patients $(42.9 \%)$, moderate in 167 patients $(47.5 \%)$, and severe in 34 patients $(9.6 \%)$. Table 1 depicts the demographic characteristics of the patient population according to the severity of AAAD. Patients with higher grades of AAAD were older and had a higher rate of hypertension and elevated creatinine levels. They exhibited more cerebrovascular disease and peripheral vascular disease and they had higher EuroSCORE and McSPI stroke risk indices. Table 2 lists the findings by EAS and palpation as well as the operative modifications used after detection of AAAD.

As shown in Table 3 the overall stroke rate was $2.3 \%$, with a difference between the 3 AAAD groups probably due to chance $(P=.935)$. No lethal stroke occurred in the patient series. Seven of the 8 patients who had a stroke were available for a follow-up interview. Four of them showed $100 \%$ activity of daily living according to the Barthel Index. 


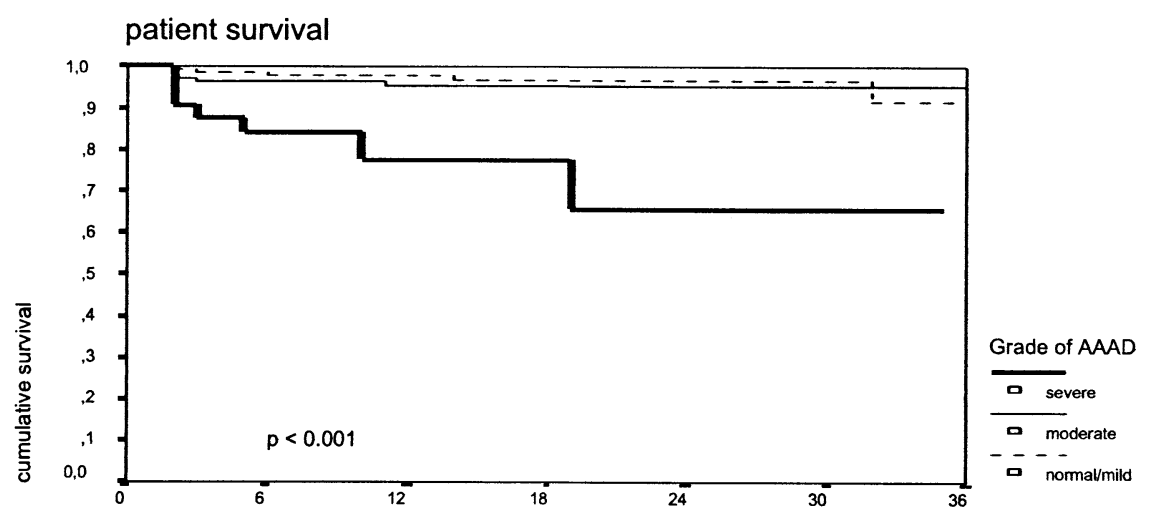

A

survival time (months)

stroke free survival

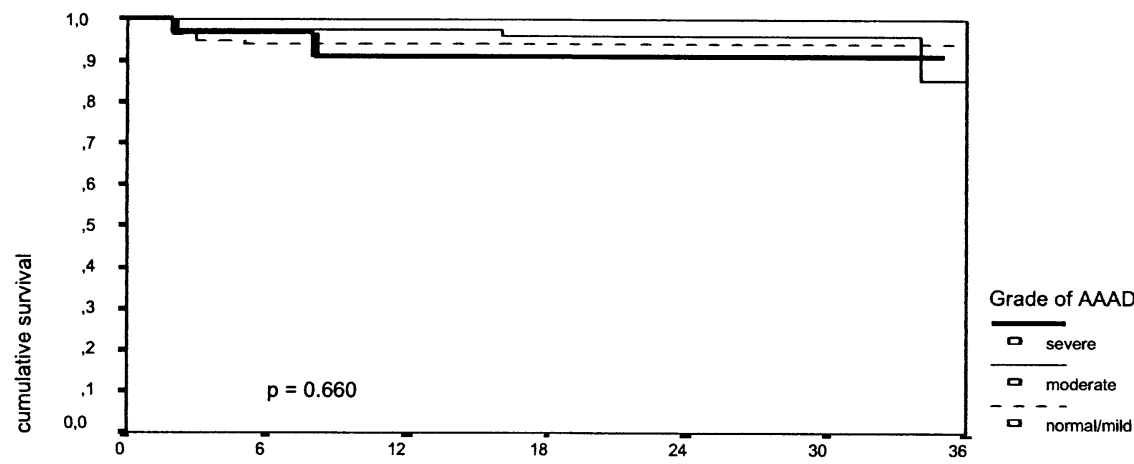

B

survival time (months)

myocardial infarction free survival

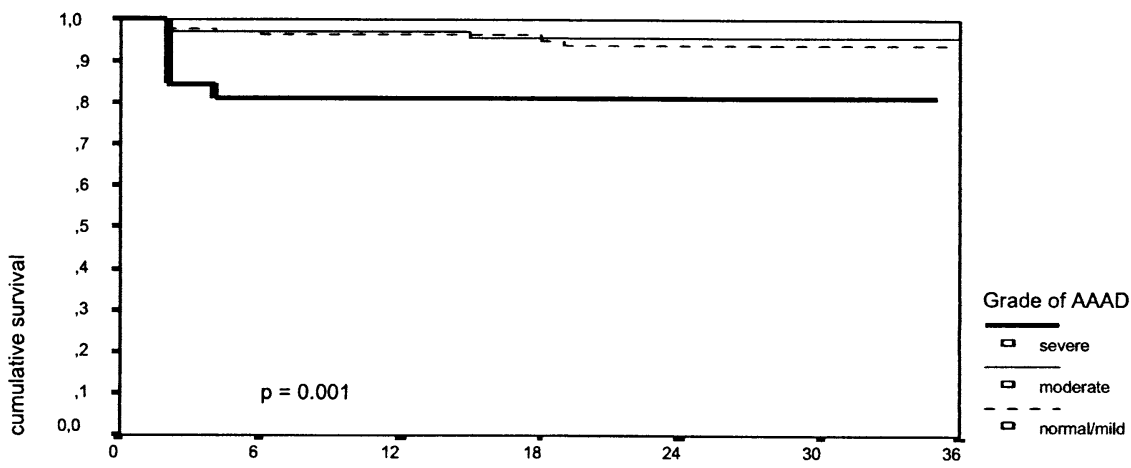

C survival time (months)

Figure 2. Kaplan-Meier plots according to the severity of ascending aortic atherosclerotic disease for patient survival (A), stroke-free survival (B), and freedom from myocardial infarction (C) during long-term follow-up (for reason of a clear arrangement, censored events and numbers at risk are not depicted).

One patient exhibited $90 \%$ activity of daily living and 2 patients exhibited $75 \%$ activity of daily living. ${ }^{13}$

Perioperative death and myocardial infarction, however, occurred more often if a higher grade of AAAD was present $(P=.005$ and $P=.001$, respectively, see Table 3$)$.
Logistic regression analyses for the perioperative end points mortality and myocardial infarction adjusting for EuroSCORE rendered the results in Table 4, with the maximal wall thickness of the ascending aorta still having an important influence on both end points. 

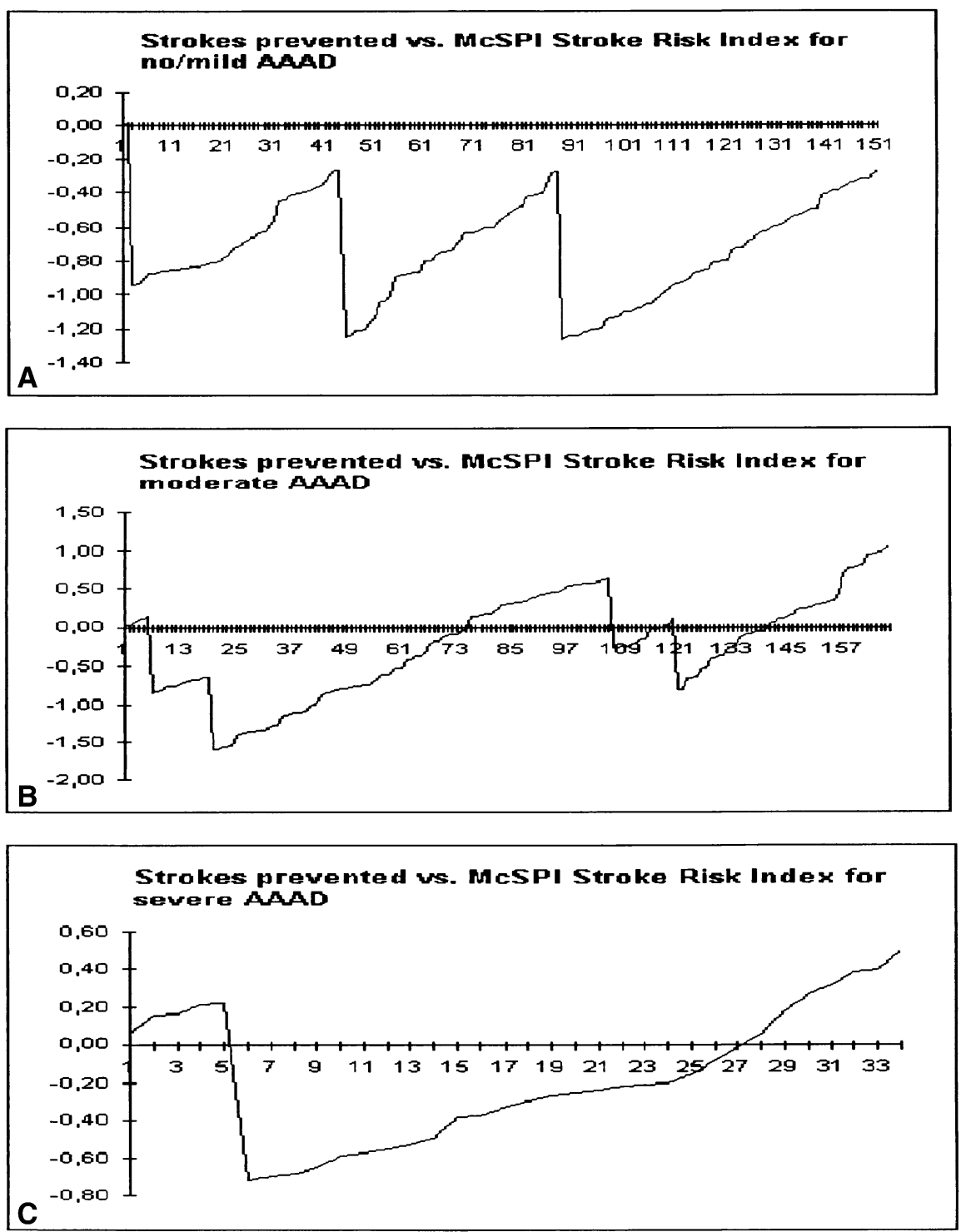

Figure 3. A, B, C, CRAS according to the severity of ascending aortic atherosclerotic disease.

The difference in the occurrence of strokes among the 3 groups during medium-term follow-up could be due to chance $(P=.660$, see Figure $2, B)$.

Medium-term survival was higher in patients with no/ mild and moderate ascending aortic disease as compared with patients having severe disease $(P<.001$, see Figure $2, A)$.

Myocardial infarction-free survival was lower in patients with severe AAAD but myocardial infarctions primarily occurred during the perioperative period $(P=.001$, Figure 2,C).

Cox proportional hazard regression analyses for the 36month end points of mortality and myocardial infarction, adjusting for EuroSCORE, rendered the results in Table 4, with the maximal wall thickness of the ascending aorta having still an important influence on medium-term survival.

CRAS with some variations corresponded with the McSPI stroke risk index predictions in all 3 patient groups (Figure 3, $A, B$, and $C$ ). Aortic no-touch techniques with extra-anatomic bypass strategies, which were used exclusively in patients with severe ascending aortic atherosclerosis, resulted in evident stroke prevention versus McSPI stroke risk index predictions, whereas this was not the case using single aortic crossclamping, which was primarily applied in moderate ascending aortic atherosclerosis (Figure 4). Marked gains in cumulative risk-adjusted mortality versus EuroSCORE predictions were ob- 

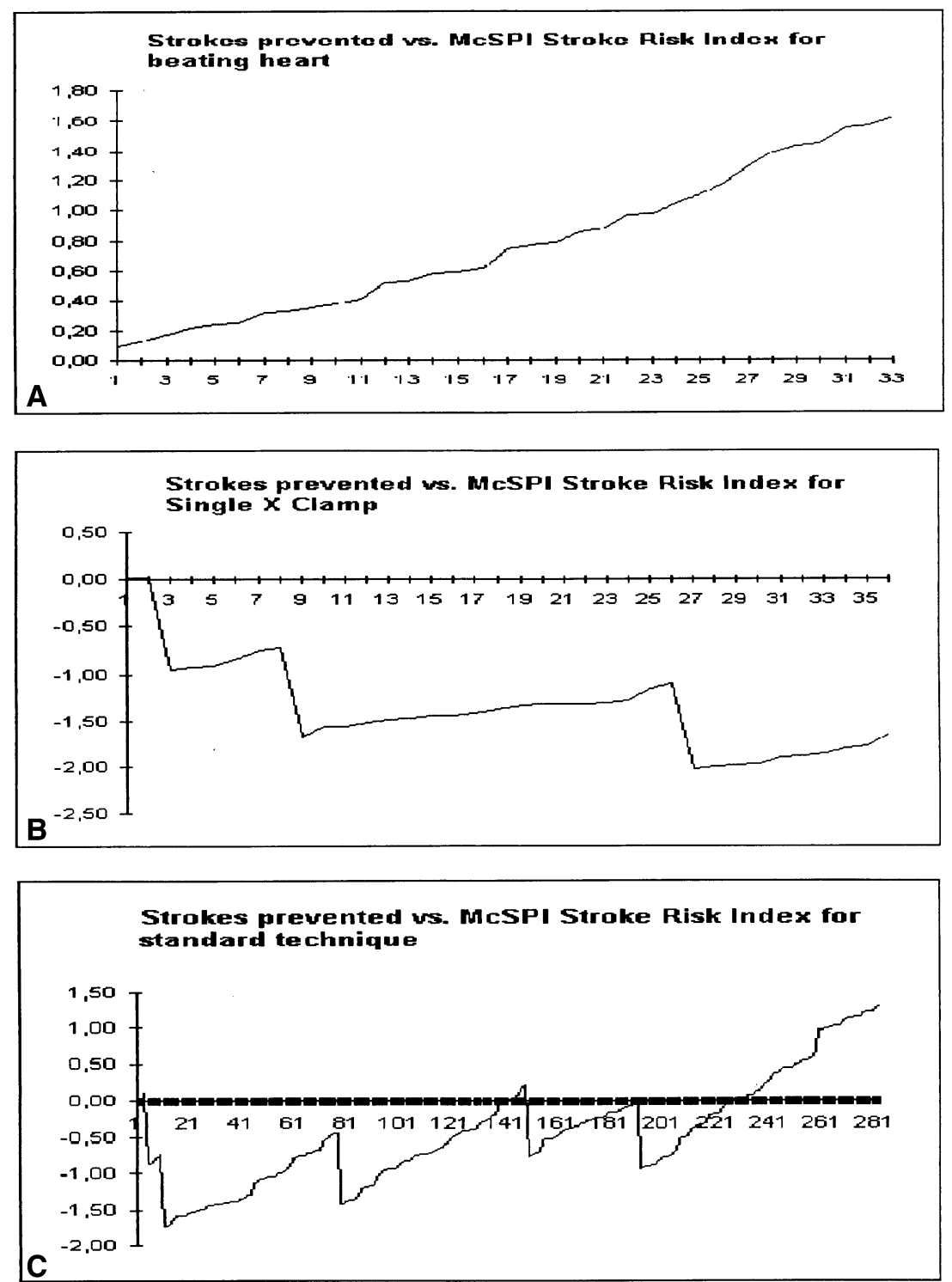

Figure 4. CRAS in patients operated on the beating heart (A), using single crossclamp technique (B), and partial occluding clamp (C).

tained in patients with no/mild and moderate AAAD, whereas losses of lives versus EuroSCORE predictions were noted in patients with severe AAAD (Figure 5, $A, B$, and $C$ ).

\section{Discussion}

In this retrospective study EAS was performed in 352 patients undergoing isolated primary CABG. Clinically important associations were found between age, cerebrovascular disease, peripheral vascular disease, EuroSCORE, McSPI stroke risk index, preoperative creatinine, hypertension, and the severity of ascending aortic atherosclerosis. Except for the EuroSCORE, McSPI stroke risk index, and preoperative creatinine, all these variables have been re- ported as being risk factors for the presence of AAAD in CABG patients. ${ }^{6,14-16}$ If not performed on a routine basis it would, in our opinion, be advisable to conduct EAS at least in the described risk groups.

Patients with severe AAAD were found to have the highest prevalence of death and myocardial infarction. With modification of the surgical technique, according to the algorithm used in this study, cerebrovascular complications could be kept at a prevalence of $2.9 \%$ in the 30-day perioperative period for high-risk patients exhibiting severe ascending aortic disease. In these patients the stroke rate predicted by the McSPI index would have been $4.4 \%$. Studies from the early 1990s have demonstrated stroke rates 

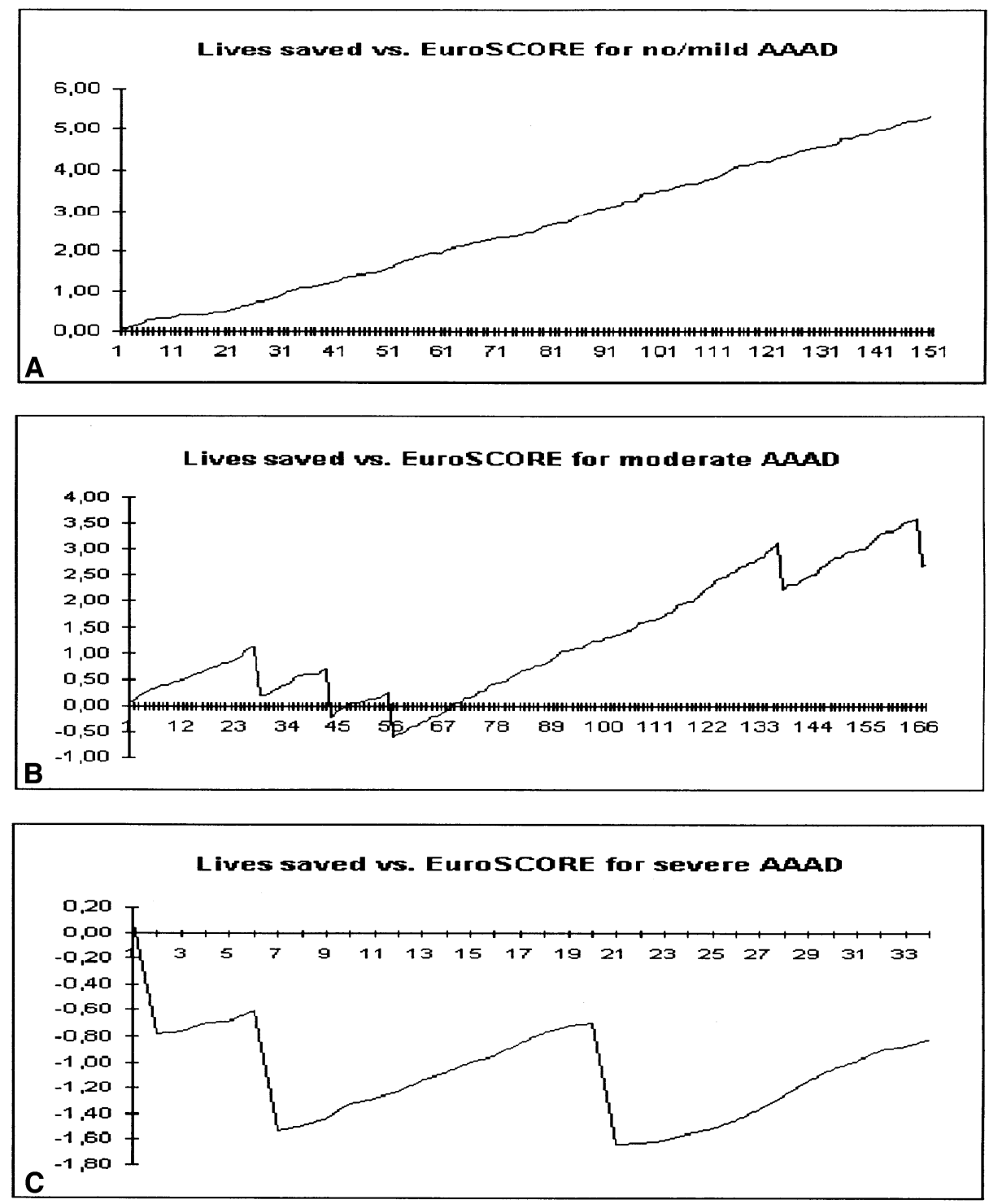

Figure 5. A, B, C, CRAM according to the severity of ascending aortic atherosclerotic disease.

in the range of $19 \%$ to $45 \%$ if a severely diseased ascending aorta is manipulated during conventional CABG. ${ }^{17,18}$ Looking at these data it seems likely that strokes could be prevented by changing the standard surgical approach. Van der Linden and colleagues ${ }^{4}$ could not show a reduction in the prevalence of postoperative strokes with minor modifications in surgical technique in a series of 921 consecutive patients undergoing cardiac surgery. The prevalence of strokes in their series was $1.8 \%$ in patients without atherosclerotic disease of the ascending aorta and $8.7 \%$ in patients with the disease despite minor surgical modifications. This may advocate a more aggressive change of the operative technique in the presence of severe AAAD. With an algorithm to support the surgical decision making when severe ascending atherosclerosis is present, the perioperative neurologic event rates could be kept well below the expected ones in our study population. In contrast to our study with patients undergoing primary isolated CABG, van der Linden's group ${ }^{4}$ investigated a more heterogeneous patient population including valve procedures and coronary reoperations, which may have a negative impact on their stroke rate. Despite the fact that the stroke reduction versus the McSPI stroke risk index predictions were not absolutely striking, it is a very satisfying fact that in our series no lethal stroke occurred and that a marked recovery potential in the patients who had a stroke was noted. According to recent data, stroke in cardiac surgery is associated with a $19 \%$ in-hospital mortality and only $25.8 \%$ of patients return to a normal level of activity. ${ }^{19}$ 
Despite attempts to completely revascularize the heart, myocardial infarction rates were higher in patients with severe AAAD, even adjusted for EuroSCORE $(P=.045$, odds ratio 1.41, confidence interval 1.01-1.98). Incomplete revascularization in some cases and predominance of diffuse coronary artery disease, as well as a possible malfunction of extra-anatomical bypass grafts and selection bias toward cardiac high-risk patients, may have been the reasons for this.

In the group of patients who underwent the beating heart and aortic no-touch concept, the stroke rate was $0 \%$, and we regard this strategy as a very promising method for patients with extensive ascending aortic disease. For comparison, Patel and colleagues ${ }^{20}$ have most recently described a near $0 \%$ stroke rate using beating heart total arterial revascularization without aortic manipulation.

Single aortic crossclamping has repeatedly been demonstrated to be a method that can prevent strokes and myocardial infarction in CABG. ${ }^{8,9}$ We applied this method primarily in patients with moderate AAAD and in 15 patients in combination with intra-aortic filtration. Complete prevention of strokes, however, was not observed with this technique in our own series.

Ascending aortic atherosclerosis was an independent predictor of stroke in previous studies. ${ }^{4,16,21}$ In our series the disease was not associated with neurologic events, possibly demonstrating a preventive effect of the surgical modifications used.

The presence of severe atherosclerosis in the ascending aorta was associated with a higher perioperative mortality and myocardial infarction rate (see Table 4). Similar mortality differences for patients with the disease have been reported in the literature. ${ }^{16,22}$ The presence of major ascending aortic atherosclerosis is a marker for increased hospital mortality and morbidity even when neurologic complications can be kept low.

The cumulative risk-adjusted mortality plots for our patients with normal aorta or moderate ascending aortic atherosclerosis demonstrate that highly satisfying results in CABG can be obtained with the use of the extracorporeal circulation when the patient group with severe ascending aortic atherosclerosis is excluded. Lives saved versus EuroSCORE predictions have so far only been demonstrated for off-pump CABG. ${ }^{23}$

Concerning 3-year survivals, our data are well comparable with results published by Moshkovitz and colleagues, ${ }^{22}$ who described a 3-year survival in the $60 \%$ range for CABG patients with calcified ascending aorta. Similar results were reported by Davila-Roman and coworkers. ${ }^{3}$ AAAD has most recently been discussed as a risk factor for long-term neurologic events. Our patients with severe AAAD did not have a higher incidence of neurologic events per 3 years, as compared with patients who exhibited moderate or mild disease (Figure 2, B). Davila-Roman and colleagues ${ }^{3}$ described differences in the occurrence of stroke between similar patient groups. When looking at his data in detail, strokes in his series primarily occurred in the perioperative period and on the long-term course there were only slight differences between patients with different grades of AAAD.

From our data we conclude that EAS in CABG grafting can lead the surgeons' decisions toward modifications of operative techniques in the presence of ascending aortic atherosclerosis. By these modifications low stroke rates can be achieved in high-risk patients with severe disease. Modifications using off-pump CABG and aortic no-touch techniques seem to play a very promising role in management of ascending aortic atherosclerosis. Probably the severity of strokes can be reduced by changes in the operative strategy. Mortality and myocardial infarction, however, may be prevalent in patients with severe ascending aortic atherosclerosis. The impact of ascending aortic atherosclerosis on medium-term cerebral events is probably less dramatic than previously reported.

\section{Limitations of the Study}

This study has its limitations because of its retrospective design; in addition, there was no uniform protocol for the operative strategy, but in fact an algorithm was used as a guideline for operative modifications in the presence of AAAD, with the individuality of the surgeon's decision. However, it would be impossible to conduct this study in a randomized prospective fashion.

\section{References}

1. Roach GW, Kanchuger M, Mangano CM, et al. Adverse cerebral outcomes after coronary bypass surgery. Multicenter Study of Perioperative Ischemia Research Group and the Ischemia Research and Education Foundation Investigators. N Engl J Med. 1996;335:1857-63.

2. Keon WJ, Heggtveit HA, Leduc J. Perioperative myocardial infarction caused by atheroembolism. J Thorac Cardiovasc Surg. 1982;84:84955 .

3. Davila-Roman VG, Murphy SF, Nickerson NJ, Kouchoukos NT, Schechtman KB, Barzilai B. Atherosclerosis of the ascending aorta is an independent predictor of long-term neurologic events and mortality. J Am Coll Cardiol. 1999;33:1308-16.

4. van der Linden J, Hadjinikolaou L, Bergman P, Lindblom D. Postoperative stroke in cardiac surgery is related to the location and extent of atherosclerotic disease in the ascending aorta. J Am Coll Cardiol. 2001;38:131-5.

5. Davila-Roman VG, Barzilai B, Wareing TH, Murphy SF, Kouchoukos NT. Intraoperative ultrasonographic evaluation of the ascending aorta in 100 consecutive patients undergoing cardiac surgery. Circulation. 1991;84:47-53.

6. Wareing TH, Davila-Roman VG, Daily BB, et al. Strategy for the reduction of stroke incidence in cardiac surgical patients. Ann Thorac Surg. 1993;55:1400-8.

7. Wilson MJ, Boyd SYN, Lisagor PG, Rubal BJ, Cohen DJ. Ascending aortic atheroma assessed intraoperatively by epiaortic and transesophageal echocardiography. Ann Thorac Surg. 2000;70:25-30.

8. Aranki SF, Rizzo RJ, Adams DH, et al. Single-clamp technique: an important adjunct to myocardial and cerebral protection in coronary operations. Ann Thorac Surg. 1994;58:296-303. 
9. Aranki SF, Sullivan TE, Cohn LH. The effect of the single aortic cross-clamp technique on cardiac and cerebral complications during coronary bypass surgery. J Card Surg. 1995;10:498-502.

10. Bonatti JO, Hangler HB, Antretter H, Müller LC. Axillocoronary bypass for severely atherosclerotic aorta in coronary bypass grafting. J Thorac Cardiovasc Surg. 1998;115:956-7.

11. Nashef SAM, Roques F, Hammil BG, et al. Validation of European System for Cardiac Operative Risk Evaluation (EuroSCORE) in North American cardiac surgery. Eur J Cardiothorac Surg. 2002; 22:101-5.

12. Newman MF, Wolman R, Kanchuger M, et al. Multicenter preoperative stroke risk index for patients undergoing coronary artery bypass graft surgery. Multicenter Study of Perioperative Ischemia (McSPI) Research Group. Circulation. 1996;94:74-80.

13. Mahoney FI, Barthel DW. Functional evaluation: the Barthel Index. Md State Med J. 1965;14:61-5.

14. Brener BJ, Brief DK, Alpert J, Goldenkranz RJ, Parsonnet V. The risk of stroke in patients with asymptomatic carotid stenosis undergoing cardiac surgery: a follow-up study. J Vasc Surg. 1987;5: 269-79.

15. Landymore R, Spencer F, Colvin S, et al. Management of the calcified aorta during myocardial revascularization. J Thorac Cardiovasc Surg. 1982;84:455-6.

16. Davila-Roman VG, Barzilai B, Wareing TH, Murphy SF, Schechtman
KB, Kouchoukos NT. Atherosclerosis of the ascending aorta: prevalence and role as an independent predictor of cerebrovascular events in cardiac patients. Stroke. 1994;25:2010-6.

17. Lynn GM, Stefanko K, Reed JF, Gee W, Nicholas G. Risk factors for stroke after coronary artery bypass. J Thorac Cardiovasc Surg. 1992; 104:1518-23.

18. Mills NL, Everson CT. Atherosclerosis of the ascending aorta and coronary artery bypass: pathology, clinical correlates, and operative management. J Thorac Cardiovasc Surg. 1991;102:546-53.

19. Salazar JD, Wityk RJ, Grega MA, et al. Stroke after cardiac surgery: short- and medium-term outcomes. Ann Thorac Surg. 2001;72:1195202.

20. Patel NV, Pullan DM, Fabri BM. Does off-pump total arterial revascularization without aortic manipulation influence neurological outcome? A study of 226 consecutive, unselected cases. Heart Surg Forum. 2001;5:28-32.

21. John R, Choudhri AF, Weinberg AD, et al. Multicenter review of preoperative risk factors for stroke after coronary artery bypass grafting. Ann Thorac Surg. 2000;69:30-5.

22. Moshkovitz Y, Paz Y, Shabtai E, et al. Predictors of early and overall outcome in coronary artery bypass without cardiopulmonary bypass. Eur J Cardiothorac Surg. 1997;12:31-9.

23. Sergeant P, de Worm E, Meyns B, Wouters P. The challenge of departmental quality control in the reengineering towards off-pump coronary artery bypass grafting. Eur J Cardiothorac Surg. 2001;538:43. 